Supplement of Atmos. Chem. Phys., 15, 11477-11499, 2015

http://www.atmos-chem-phys.net/15/11477/2015/

doi:10.5194/acp-15-11477-2015-supplement

(C) Author(s) 2015. CC Attribution 3.0 License.

(c) (1)

Supplement of

\title{
Transport pathways of peroxyacetyl nitrate in the upper troposphere and lower stratosphere from different monsoon systems during the summer monsoon season
}

S. Fadnavis et al.

Correspondence to: S. Fadnavis (suvarna@ tropmet.res.in)

The copyright of individual parts of the supplement might differ from the CC-BY 3.0 licence. 


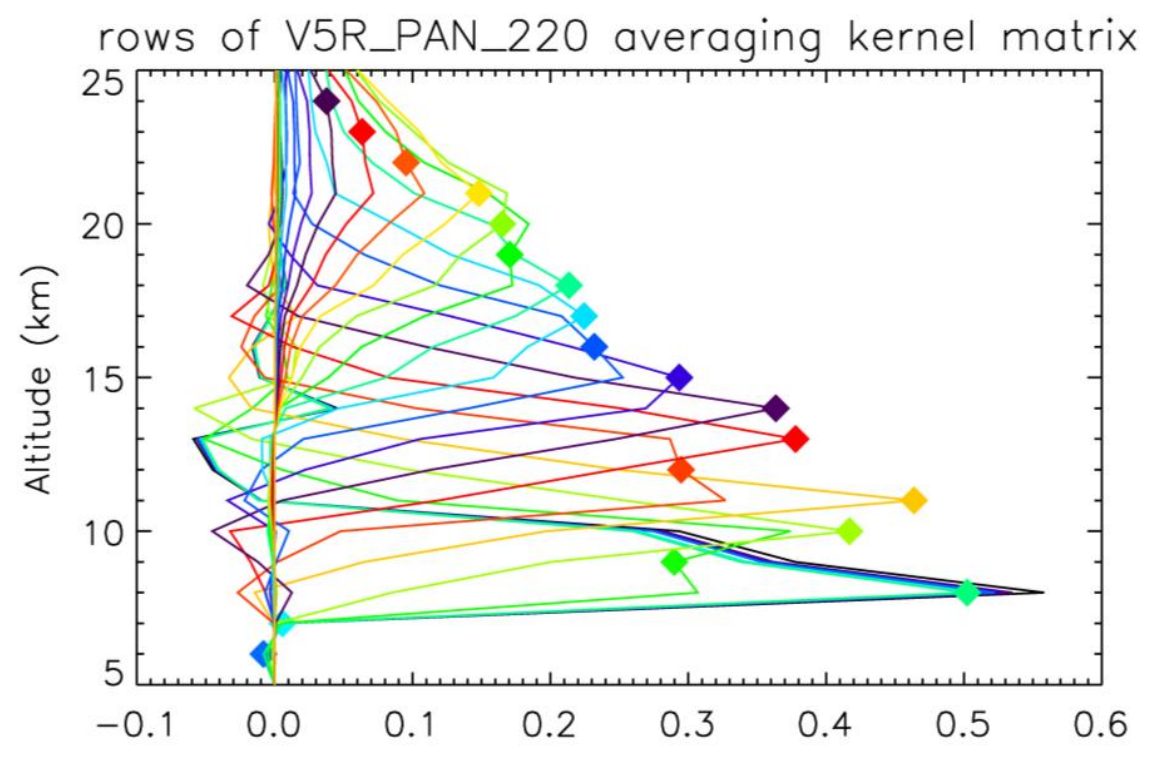

Figure S1: Averaging kernel rows of data version V5R_PAN_220 at the location $28^{\mathrm{O}} \mathrm{N}$ and $85^{\mathrm{O}}$ E. Diamonds indicate the respective nominal altitudes of the retrieval grid. 

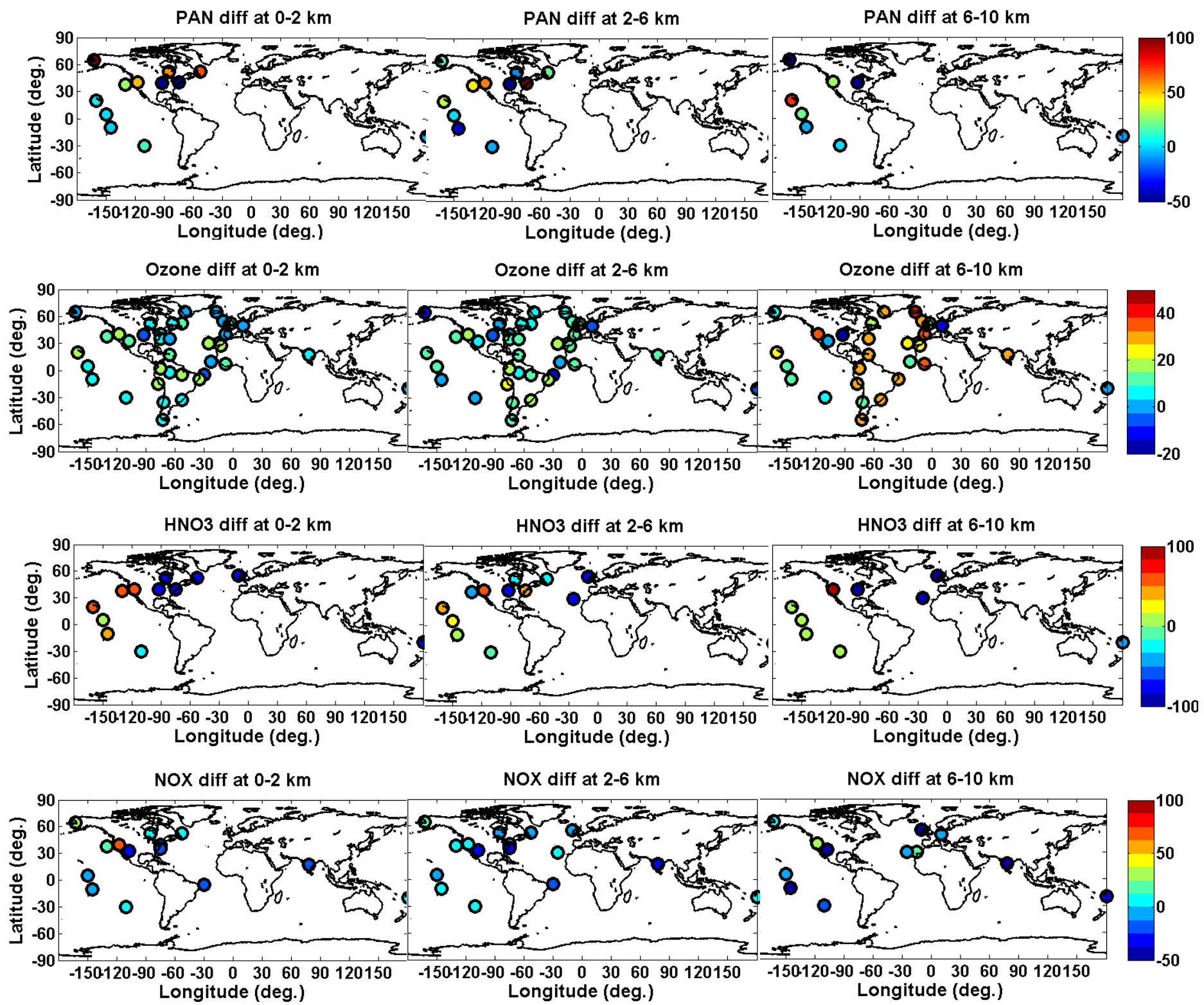

Figure S2: Global distribution of bias (ECHAM5-HAMMOZ - aircraft observations) in (PAN (ppt), ozone (ppb), $\mathrm{HNO}_{3}(\mathrm{ppt}), \mathrm{NO}_{\mathrm{x}}(\mathrm{ppt})$, for monsoon season and altitude ranges a 0 $2 \mathrm{~km} .2-6 \mathrm{~km}, 6-10 \mathrm{~km}$. 

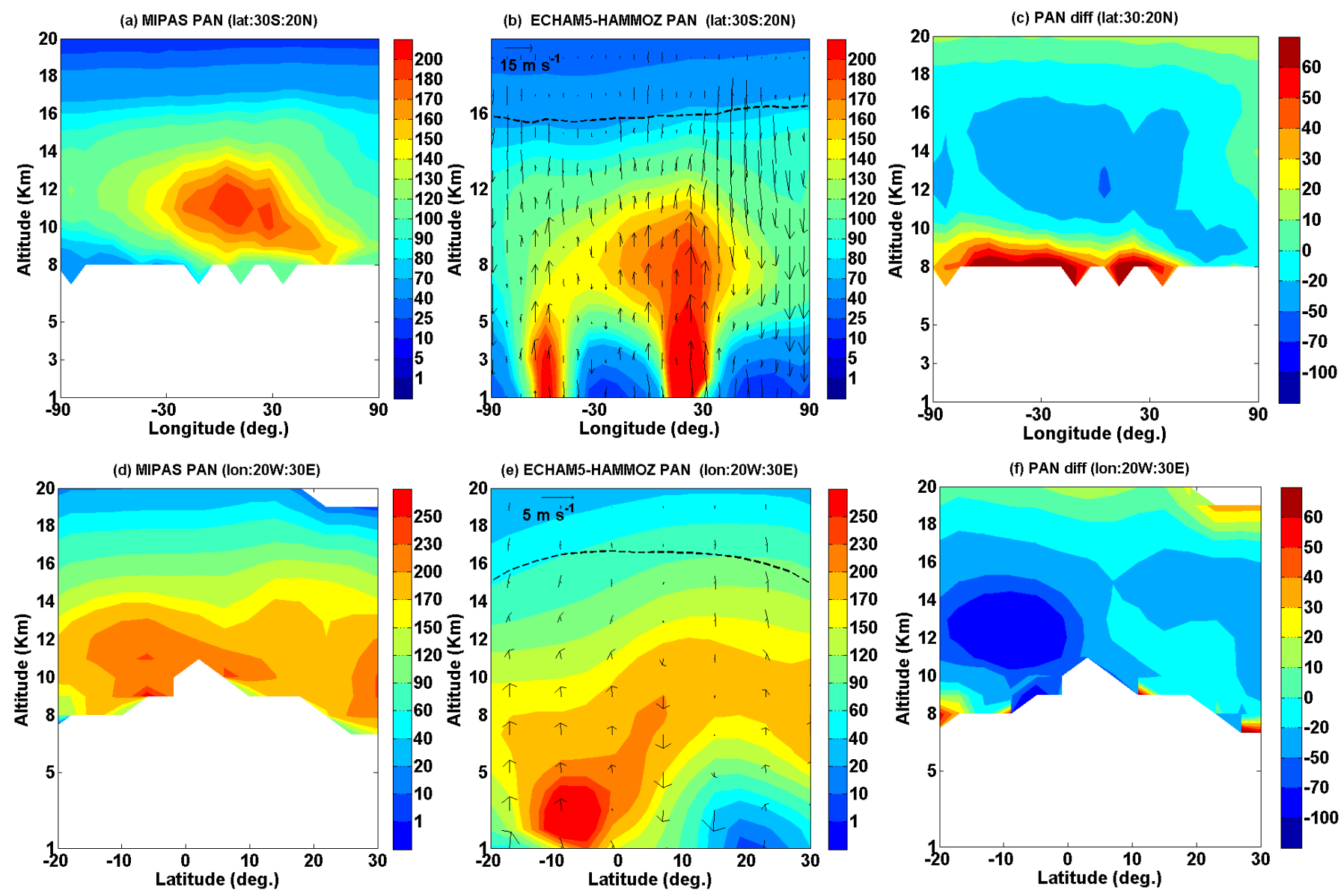

Figure S3. Longitude -altitude cross-section of PAN (ppt) averaged for monsoon season and over $30^{\circ} \mathrm{S}-20^{\circ} \mathrm{N}$ (a) MIPAS-E climatology (b) ECHAM5-HAMMOZ CTRL simulations (c) difference in PAN (ppt) (ECHAM5-HAMMOZ-MIPAS). Latitudealtitude cross-section of PAN (ppt) averaged for monsoon season and over $20^{\circ} \mathrm{W}-30^{\circ} \mathrm{E}$ (d) MIPAS-E climatology (e) ECHAM5-HAMMOZ CTRL simulations (f) difference in PAN (ppt) (MIPAS-ECHAM5-HAMMOZ). 
(a) ECHAM5-HAMMOZ-MIPAS diff at $13 \mathrm{~km}$

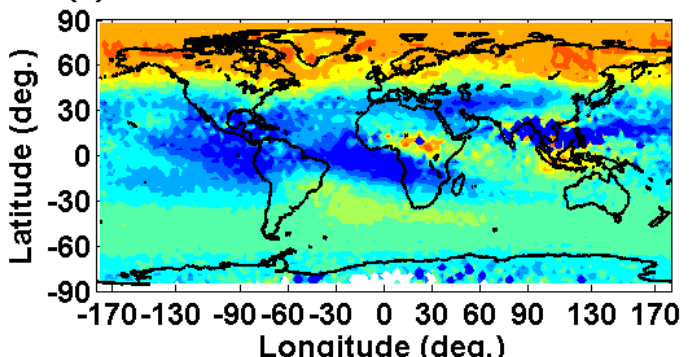

(c) ECHAM5-HAMMOZ-MIPAS diff at $17 \mathrm{~km}$

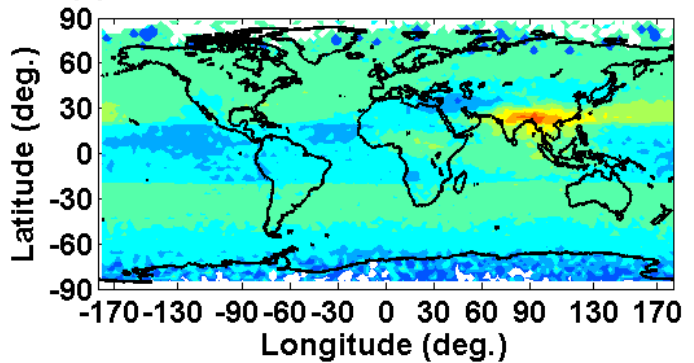

(b) ECHAM5-HAMMOZ-MIPAS diff at $15 \mathrm{~km}$
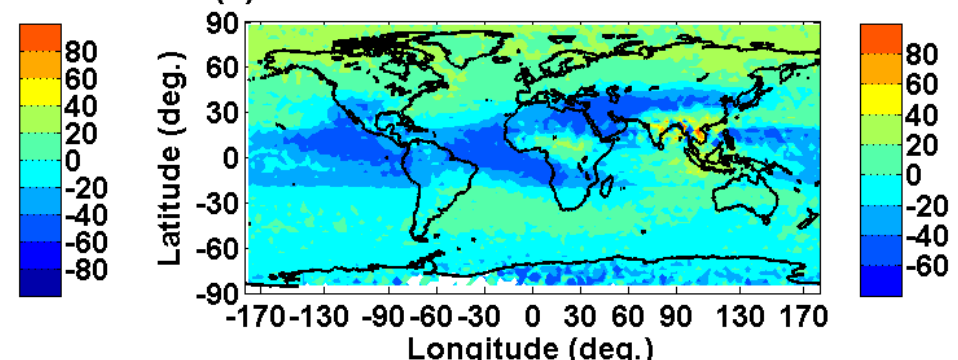

(d) ECHAM5-HAMMOZ-MIPAS diff at $20 \mathrm{~km}$

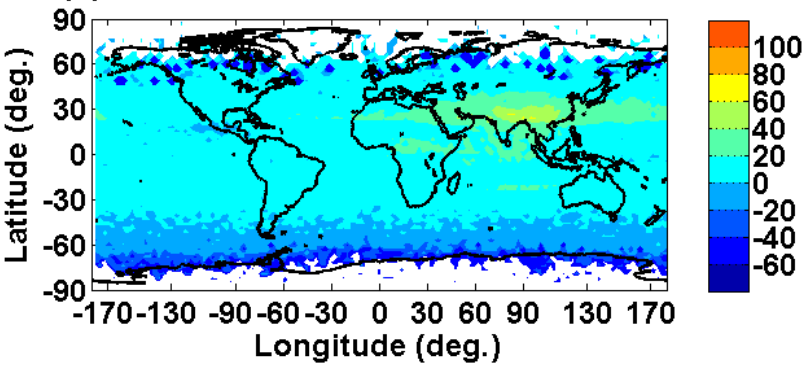

Figure S4: Differences between MIAPS observations (climatology 2002-2011) and ECHAM5-HAMMOZ reference simulation in PAN (ppt) averaged for the monsoon season (a) at $13 \mathrm{~km}$ (b) $15 \mathrm{~km}$ (c) $17 \mathrm{~km}$ and (d) $20 \mathrm{~km}$. 

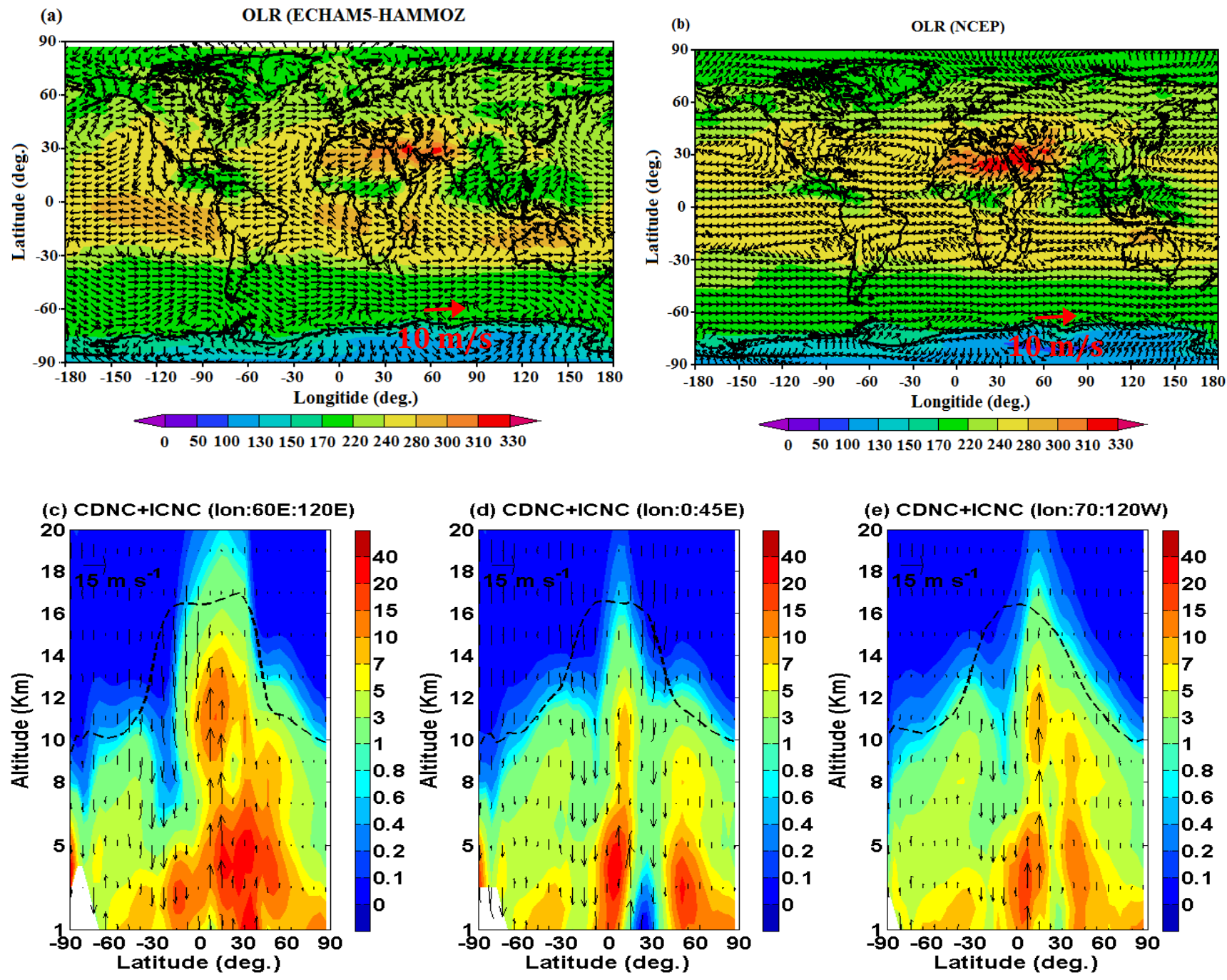

Figure S5. Distribution of simulated (a) Outgoing long wave radiations (OLR) and 850 hpa winds averaged for June-September (2000-2010), (a) ECHAM5-HAMMOZ NCEP-Reanalysis data, ECHAM5-HAMMOZ simulated cloud droplet number concentration (CDNC) and ice crystal number concentration (ICNC) (1 mg-1 ) averaged for June-September (2000-2010) (c) zonal average between $60-120^{\circ} \mathrm{E}$ and (d) zonal average between $70 \mathrm{~W}$ and $120^{\circ} \mathrm{E}$ (e) zonal average between $0-45^{\circ}$ E. The black arrows indicate wind vectors. In figures (c)-(e) the vertical velocity field has been scaled by 300 . 
(a) ECHAM5-HAMMOZ NOX (IOn:60:120E)

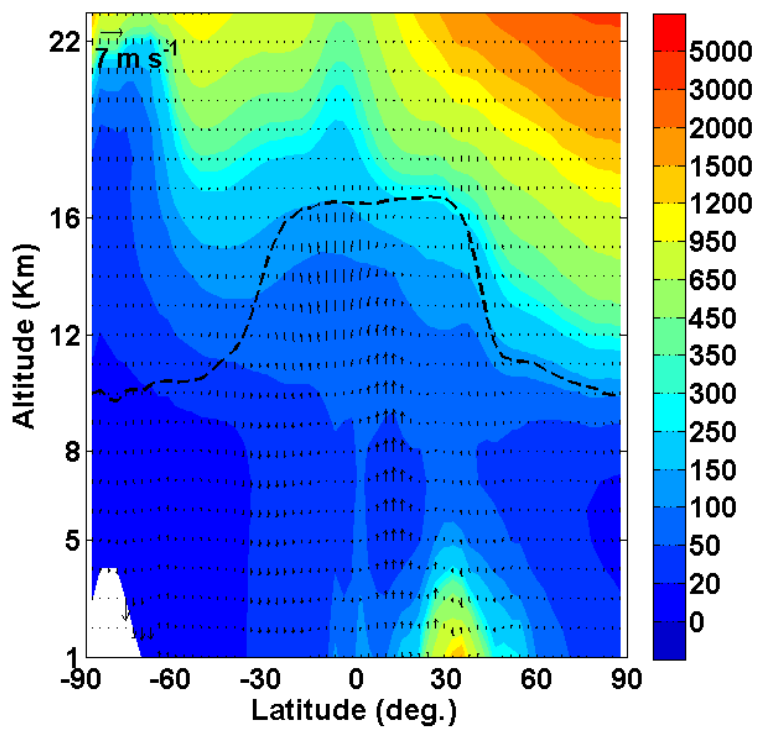

(b) ECHAM5-HAMMOZ NOX (lat:10:40N)

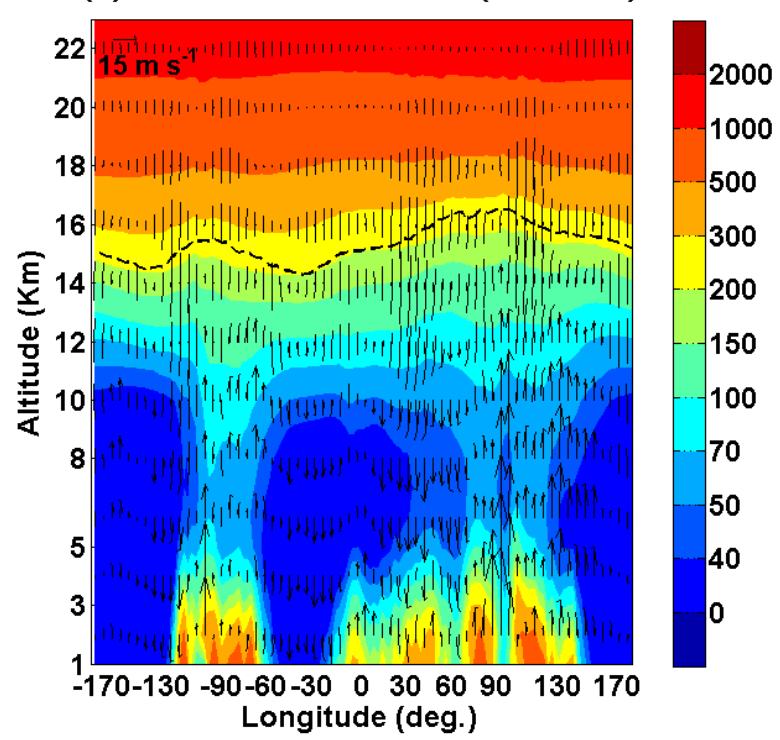

Figure S6. Seasonal mean ECHAM5-HAMMOZ $\mathrm{NO}_{\mathrm{x}}(\mathrm{ppt})$ obtained from reference simulation (a) Latitude -pressure cross section averaged over $60^{\circ} \mathrm{E}-120^{\mathrm{O}} \mathrm{E}$ (b) Longitude-pressure cross section averaged over $10^{\mathrm{O}} \mathrm{N}-40^{\mathrm{O}} \mathrm{N}$. The black arrows indicate wind vectors. The vertical velocity field has been scaled by 300 . 